\title{
In vivo veritas, in vitro-not so sure when it comes to ARTs
}

\author{
David F. Albertini ${ }^{1}$
}

Published online: 15 March 2019

(C) Springer Science+Business Media, LLC, part of Springer Nature 2019

There may be something to the ancient adage that in wine there is truth (in vino veritas), but when it comes to so many of the ex vivo manipulations we subject complex cells, tissues, and organs to, the line of distinction between reality and artifact becomes blurred. So much of the backdrop to ARTs as they are currently practiced derived from decades of work on mammalian gametes and embryos managed as they were in the only experimentally tractable way possible-EX VIVO. Consonant with reductionist trends of the times, to isolate, purify, concentrate, and analyze, became the mantra of scientific disciplines seeking answers to basic questions clouded by the compounding variables that made the organ or organism work. In these days of regenerative medicine, and in the context of all strategies "personalized," putting the pieces of the puzzle back together under the guise of quasi functioning units (sic organoids, artificial organs) is a charge engaged by many an investigator that would like to add "reconstitution" to their designated mantra.

This month in JARG, we deliver an assortment of articles some of which span the matter of in vitro manipulations in the everyday practice of human ARTs. And some of which exemplify where we are with stem cell rejuvenation of organs like the aging ovary or the fine tuning of embryo selection after extended culture, with the latter increasingly normative in clinical circles.

While long sought after for a variety of conditions positioned within the spectrum from oocyte retrieval to and through embryo transfer, operational in vitro maturation (IVM) of human oocytes has yet to be anointed as an official ART add-on in our menu of options. The reasons are manifold not the least of which is the lack of compelling evidence to support the deployment of a technique that remains

David F. Albertini

eicjarg@gmail.com

1 Center for Human Reproduction, New York, NY, USA substandard with respect to live birth rates. But a step back in history reminds us that IVM holds a special distinction in the annals of human IVF. Try contextualizing the following:

\section{Dozens of eggs were cultured. I examined them eagerly after three, six, nine, and twelve hours. None of them changed their appearance in any way whatsoever. Their unwinking nuclei gazed back at me steadily whether they had come from rhesus monkeys, baboons, or from humans. They would not ripen, their chromosomes marched nowhere at all, no matter which culture medi- um I used.}

If you guessed this was some person frustrated with achieving mammalian oocyte IVM somewhere in the distant past, well, you are correct. The "unwinking nuclei" that we now refer to as germinal vesicle-stage oocytes was the descriptor Bob Edwards used in his pioneering attempts to not simply make IVM work but to satisfy his primordial curiosity regarding oocyte chromosomes, which he desperately wanted to visualize. This passage is taken from the book by Edwards and Steptoe, "A Matter of Life: The story of a medical breakthrough" (William Morrow and Company, Inc., New York, 1980), and admittedly represents yet another example of the determination Edwards exhibited in his quest for truth.

Deploying the microscopic diligence of a van Leuwenhoek, Edwards did finally achieve IVM as we know it today and popularized an in vitro manipulation that has been foundational in the field of reproductive biology for the past five decades [1]. Among the more notable, but not necessarily original, observations he made was that mammalian oocytes generally will resume and complete their maturation from "unwinking nuclei" to metaphase-2 once released from the ovarian follicle suggesting the presence of an intrinsic inhibitory mechanism inactivated naturally in response to LHinduced ovulation.

We now know much of the biochemical and physiological underpinnings involved with the regulation of oocyte maturation. Gene expression changes in cumulus cells, and an altered 
wiring system between the oocyte and cumulus cells orchestrate a sophisticated system of signals and responses that initiate and support the various steps of oocyte maturation [2]. Once propagated from cumulus to the oocyte, an equally impressive array of molecular adjustments are made to resident RNA, protein, and organelle species within the oocyte that not only propel the cell cycle (so-called nuclear maturation) but prepare the oocyte cytoplasm for the many tasks that will have to be engaged in order for fertilization and preimplantation development to occur (so-called cytoplasmic maturation) [3]. Despite this wealth of knowledge, too many of our patients yield oocytes that are immature and for these patients, few alternatives exist, as a result of advancing age, of poor ovarian responsiveness, or for reasons that have escaped us.

The bovine model has been one of the most useful animal models for improving IVM technology. While strategies have been developed in the bovine system for optimizing IVM, this kind of research also serves to warn practitioners of limitations inherent to the overall quality of IVM oocytes, and their resultant embryos with concerns raised specifically with regard to the overall length of oocyte culture and untoward epigenetic consequences [4]. This month, Buratini and Comizzoli summarize the results from several papers in this issue that bring the reality of IVM a step closer to the clinical laboratory. These articles provide a status report on current strategies using the bovine model and begin to narrow down the list of cumulus genes whose patterns of expression may finally define biomarkers of use in establishing the likelihood of live births resulting from conventional stimulation cycles.

Although the number of molecular players identified in either the oocyte or cumulus compartments continues to grow [5], plotting a successful strategy for human oocyte IVM will require a deep understanding of the communication pathways mediating the conversation between cumulus and oocyte prior to and in response to an LH surge [6]. At the heart of achieving synchronized maturation in vitro or defining sentinels in cumulus predictive of embryo quality, the matter of timing in response to trigger from onset to polar body emission should not be underestimated and is challenging to monitor in an everyday ART laboratory doing multiple procedures.
Towards this end, we draw attention to the paper by Holubcova and her colleagues who set criteria that allow embryologists to perform ICSI at a time that reflects the optimum for a given oocyte (Egg maturity assessment prior to ICSI prevents premature fertilization of late-maturing oocytes; https://doi.org/10.1007/s10815-018-1393). This study provides important insights into the problem of timing for fertilization and deals with patients whose sensitivity to gonadotropin stimulation is poor or variable likely contributing to abnormal fertilization or development due solely to the heterogeneous nature of in vivo maturation.

Next month, we will take our readership on a ride back into the future of PGT. With pause, we celebrate the laboratories that have finally designed and completed studies that will give our field unbiased direction for the application of genetic testing modalities so that future recommendations can draw upon a framework of high-quality evidence. Stay tuned.

\section{References}

1. Edwards RG. Maturation in vitro of mouse, sheep, cow, pig, rhesus monkey and human ovarian oocytes. Nature. 1965;208(5008):34951.

2. Brown HM, Dunning KR, Sutton-McDowall M, Gilchrist RB, Thompson JG, Russell DL. Failure to launch: aberrant cumulus gene expression during oocyte in vitro maturation. Reproduction. 2017;153(3):R109-R20.

3. Conti M, Franciosi F. Acquisition of oocyte competence to develop as an embryo: integrated nuclear and cytoplasmic events. Hum Reprod Update. 2018;24(3):245-66.

4. Heinzmann J, Mattern F, Aldag P, Bernal-Ulloa SM, Schneider T, Haaf T, et al. Extended in vitro maturation affects gene expression and DNA methylation in bovine oocytes. Mol Hum Reprod. 2015;21(10):770-82.

5. Uhde K, van Tol HTA, Stout TAE, Roelen BAJ. MicroRNA expression in bovine cumulus cells in relation to oocyte quality. Noncoding RNA. 2017;3(1).

6. Li HJ, Sutton-McDowall ML, Wang X, Sugimura S, Thompson JG, Gilchrist RB. Extending prematuration with cAMP modulators enhances the cumulus contribution to oocyte antioxidant defence and oocyte quality via gap junctions. Hum Reprod. 2016;31(4):810-21.

Publisher's note Springer Nature remains neutral with regard to jurisdictional claims in published maps and institutional affiliations. 